\title{
RECURSIVE LINEAR ORDERS WITH INCOMPLETE SUCCESSIVITIES
}

\author{
RODNEY G. DOWNEY AND MICHAEL F. MOSES
}

\begin{abstract}
A recursive linear order is said to have intrinsically complete successivities if, in every recursive copy, the successivities form a complete set. We show (Theorem 1) that there is a recursive linear order with intrinsically complete successivities but (Theorem 2) that this cannot be a discrete linear oder. We investigate the related issues of intrinsically non-low and non-semilow successivities in discrete linear orders. We show also (Theorem 3 ) that no recursive linear order has intrinsically $w t t$-complete successivities.
\end{abstract}

\section{INTRODUCTION}

In an addendum to [10] Remmel suggests that every recursive Boolean algebra with infinitely many atoms has a recursive copy whose set of atoms is incomplete. The result remains a conjecture. The corresponding result for linear orders is that every recursive linear oder has a recursive copy whose set of successivities is incomplete. (A successivity is a pair of adjacent elements.) We show in Theorem 1 that this is not true. Our proof uses a construction involving the novel idea of "separators" from Jockusch and Soare [8]. From initial wayward attempts to prove the converse to Theorem 1, we were able to salvage Theorem 2: every discrete linear oder has a recursive copy whose set of successivities is incomplete, and Theorem 3: every recursive linear order has a recursive copy whose set of successivities is $w t t$-incomplete. Theorems 1,2 , and 3 are presented in $\S \S 1,2$, and 3 , respectively. In $\S 2$ we also present three results noting some of the peculiarities of discrete linear orders: there is a discrete recursive linear order none of whose recursive copies has low successivities; every discrete recursive linear order has a recursive copy with semi-low successivities; and every semi-low $\Pi_{1}$ discrete linear oder has a recursive copy.

Our terminology and notation are as presented in Soare [14] for general recursion theory and Rosenstein [12] for recursive linear orders. A linear order is discrete if every element has an immediate predecessor and an immediate successor. It is recursive if its universe is $\omega$ (equivalently an r.e. subset of $\omega$ ) and it has a recursive order relation. We use $\mathfrak{A}, \mathfrak{L}$ and in Theorem $1, \mathscr{A}, \mathscr{B}$ to

1980 Mathematics Subject Classification (1985 Revision). Primary 06A05, 03 D45.

The authors wish to express their thanks to the referee for many helpful suggestions.

The second author was supported by a V. U. W. Postdoctoral Fellowship in the Department of Mathematics during the preparation of this paper. 
denote recursive linear orders and $\mathfrak{A}_{s}$ for the finite suborder of $\mathfrak{A}$ enumerated by stage $s$. The subscript $s$ is used exclusively to denote the finite part of an r.e. set enumerated by stage $s$. A $\Pi_{1}$ linear order is the suborder defined by a $\Pi_{1}$ subset of the recursive universe of $Q$, the recursively unique recursive linear order of type $\eta . \Pi_{n}$ linear orders are defined similarly. Since every recursive linear order is recursively isomorphic to a recursive suborder of $Q$, and conversely (see Lemma 16.3 of Rosenstein [12]), a linear order is $\Pi_{n}$ if and only if it is the suborder defined by a $\Pi_{n}$ subset of some recursive linear order. $\Pi_{n}$ linear orders may alternatively be defined as structures with a recursive universe and a $\Sigma_{n}$ order relation. That these definitions coincide is shown in Roy and Watnick [13, Corollary 4.3]. The successivity relation $S(x, y)$ is satisfied by every pair $(a, b)$ with the interval $(a, b)$ empty. The block relation $B(x, y)$ is satisfied by every pair $(a, b)$ with the interval $(a, b)$ finite or empty. Notice that, in a recursive linear order, $S(x, y)$ is a $\Pi_{1}$ relation and $B(x, y)$ is $\Sigma_{2}$. (In Moses [9, Theorem 2.2.1] it is shown that every recursive linear order with $B(x, y)$ recursive has a recursive copy with $S(x, y)$ recursive.) A block is an equivalence class under $B(x, y)$; this is the $\mathbf{c}_{F}[x]$ of Chapter 4.2 of Rosenstein [12]. We sometimes use the phrase "complete block" to stress that the elements outside a block have infinitely many elements between them and the block. A choice set consists of precisely one element from each block. Every recursive linear order has a $\Pi_{2}$ choice set $C$ defined by

$$
x \in C \Leftrightarrow \forall y<x \neg B(x, y) \quad \text { (here }<\text { is that of } \omega) .
$$

We construct a linear order $\mathfrak{A}$ as the limit of a series of finite linear orders $\mathfrak{A}_{0} \subseteq \mathfrak{A}_{1} \subseteq \mathfrak{A}_{2} \subseteq \cdots$. We consider $S(\mathfrak{A})$, the set of successivities of $\mathfrak{A}$, to be a subset of the indices of $\bar{s}_{0}, \bar{s}_{2}, \bar{s}_{2}, \ldots$, some fixed enumeration of $\omega^{2}$. Thus $i \in S(\mathfrak{A})$ denotes that $\bar{S}_{i}$ is a successivity in $\mathfrak{A}$. Note that neither $S\left(\mathfrak{A}_{s}\right) \subseteq S\left(\mathfrak{A}_{s+1}\right)$ nor $S\left(\mathfrak{A}_{s+1}\right) \subseteq S\left(\mathfrak{A}_{s}\right)$ need be true. We use the same symbol to denote an r.e. set and its characteristic function; the context will make clear which is intended.

\section{INTRINSICALLY COMPLETE SUCCESSIVITIES}

The class of recursive linear orders with intrinsically complete successivities is nonempty. This is proved in Theorem 1 via a technique which we believe lends itself to the analysis of other questions regarding the existence of recursive structures with intrinsically nonrecursive properties (see the discussion after the proof of Theorem 1). The technique was suggested to us by Jockusch and Soare [8].

Theorem 1. There is a recursive linear order $\mathfrak{A}$ with $S(\mathfrak{A})$ intrinsically complete. In fact, for each r.e. nonrecursive set $C$, there is a recursive linear order $\mathfrak{A}$ with $S(\mathfrak{A}) \equiv_{T} C$ and $S(\mathfrak{L}) \geq_{T} C$ for every recursive $\mathfrak{L}$ (clasically) isomorphic to $\mathfrak{A}$. Proof. Our construction uses an elegant idea of Jockusch and Soare [8] to diagonalize over a list $\mathfrak{L}_{0}, \mathfrak{L}_{1}, \mathfrak{L}_{2}, \ldots$ of all candidates for recursive linear orders. 
Such a list may be generated by, for example, considering $W_{e} \subseteq \omega^{2}$ to be the order relation of $\mathfrak{L}_{c}$. A $\varnothing^{\prime \prime}$-priority argument is applied to each $\mathfrak{L}_{e}$ to produce a recursive linear order $\mathscr{A}_{e}$. $\mathfrak{A}$ will be $\mathscr{S}_{0}+\mathscr{A}_{0}+\mathscr{S}_{1}+\mathscr{A}_{1}+\cdots$, where each separator $\mathscr{S}_{i}$ is a block of length $i+3$. Each $\mathscr{A}_{i}$ will have order-type $\eta$ or $(\eta+2+\eta) \cdot \tau$ for some $\tau$. Note that our separators, being blocks of size at least 3 , are distinguishable from the blocks of the $\mathscr{A}_{i}$. It follows that if $\mathfrak{L}_{c} \cong \mathfrak{A}$, then $\mathfrak{L}_{e}$ must also be of the form $\mathscr{S}_{0}^{\prime}+\mathscr{B}_{0}+\mathscr{S}_{1}^{\prime}+\mathscr{B}_{1}+\cdots$, with each $\mathscr{B}_{i} \cong \mathscr{A}_{i}$ (and $\left.\mathscr{S}_{i}^{\prime} \cong \mathscr{S}_{i}\right)$. We attempt to arrange that $\mathscr{B}_{e} \not \mathscr{A}_{e}$, thus ensuring that $\mathfrak{L}_{e} \not \mathfrak{A}$. This attempt will fail only if $S\left(\mathfrak{L}_{e}\right) \geq_{T} C$. The construction of each such $\mathscr{A}_{e}$ will be governed by the enumeration of the suborder $\mathscr{B}_{e}$ of $\mathfrak{L}_{e}$; this is where the $\Pi_{2}$ guessing comes in: If $\mathfrak{L}_{e}$ were to be isomorphic to $\mathfrak{A}$, then $\mathscr{B}_{e}$ would be definable as the interval between the separators $\mathscr{S}_{e}^{\prime}$ and $\mathscr{S}_{e+1}^{\prime}$-(complete) blocks of size $e+3$ and $e+4$, respectively. These separators, and consequently $\mathscr{B}_{e}$, are definable in $\mathfrak{L}_{e}$ by $\Pi_{e}$ formulae. Our strategy against $\mathfrak{L}_{e}$ is to begin with an ordered list of pairs of blocks $\left(\bar{n}_{1}, \bar{n}_{2}\right)$ of $\mathfrak{L}_{e}$ of size $e+3$ and $e+4$, respectively-candidates for the pair of separators $\left(\mathscr{S}_{e}^{\prime}, \mathscr{S}_{e+1}^{\prime}\right)$. We use $\Pi_{2}$ guessing to settle on the "correct" pair, thus obtaining a recursive enumeration of $\mathscr{B}_{e}$, and then construct $\mathscr{A}_{e} \not \mathscr{B}_{e}$. We will describe this $\Pi_{2}$ strategy later; for now we describe the basic module, under the assumption that we know the pair $\left(\mathscr{S}_{e}^{\prime}, \mathscr{S}_{e+1}^{\prime}\right)$ and the recursive linear order $\mathscr{B}_{e}$.

Given a recursive linear order $\mathscr{B}$ we construct a recursive linear order $\mathscr{A}$ such that either $\mathscr{B} \not \mathscr{A}$ or $\mathscr{S}(\mathscr{B}) \geq_{T} C$. $\mathscr{A}$ will have order-type $(\eta+2+\eta) \cdot \tau$ for some nonempty $\tau$ and $\mathscr{S}(\mathscr{A})$ will be $\equiv_{T} C$. If $\mathscr{S}(\mathscr{B})$ happens to be infinite, $\tau$ will be one of $\omega+\omega^{*}, \omega+k$, or $k+\omega^{*}$ for some finite (or empty) $k$. We will build $\mathscr{A}$ anticipating this possibility and write $L$ and $R$ for the two "blocks" of successivities of $\mathscr{A}$ defined by the blocks of $\tau$; i.e. $L$ contains all those successivities of $\mathscr{A}$ that have only finitely many successivities to their left and $R$ those with only finitely many to their right. At each stage $s$ we will define $L_{s}$ and $R_{s}$, approximations to $L$ and $R$. Some of the successivities of $\mathscr{A}_{s}$ will be labelled; these labels may be subsequently removed. At stage $s+1$ we add points to $\mathscr{A}_{s}$, one at the extreme left, one at the extreme right and one between every unlabelled successivity. Thus a successivity in $\mathscr{A}_{s}$ will remain one in $\mathscr{A}_{s+1}$ if and only if it is labelled. Those that retain their labels forever will be the successivities of $\mathscr{A}$. Successivities introduced to the extreme left or right of $\mathscr{A}$ will never be labelled; consequently the separators $\mathscr{S}_{i}$ will not merge with these $\mathscr{A}$.

At stage $s$ we define the partial function $f_{s}$ by $f_{s}(n)=j$ if and only if $\bar{s}_{j}$ is the $(n+1)$ st element of $\mathscr{S}\left(\mathscr{B}_{s}\right)$ (as a subset of, and with order inherited from, the list $\left.\omega^{2}\right) . f_{s}(n)$ is undefined if $\left|\mathscr{S}\left(\mathscr{B}_{s}\right)\right|<n+1$, i.e. if $\left|\mathscr{B}_{s}\right|<$ $n+2$. If $\mathscr{S}(\mathscr{B})$ is infinite, then $f=\lim _{s} f_{s}$ exists and is a total function, and $\mathscr{S}(\mathscr{B}) \geq_{T} f$. We arrange in this case either that $\mathscr{B} ¥ \mathscr{A}$ or that $f \geq_{T} C$ and therefore $\mathscr{S}(\mathscr{B}) \geq_{T} C$. If $\mathscr{S}(\mathscr{B})$ is finite, then, since $\mathscr{S}(\mathscr{A})$ is not, we have $\mathscr{B} \not \mathscr{A}$. 


\section{THE CONSTRUCTION}

Begin with $\mathscr{A}_{0}=\varnothing$. At each further stage $s$, let $k=C_{s}-C_{s-1}$ (we arrange that a single element of $C$ is enumerated at each stage), and perform the following four steps:

1. Remove all labels $\geq k$.

2. If $m<k$ and there is no successivity labelled $m$, introduce one, placing it immediately to the right of $L_{s-1}$. These new successivities may be placed in any order between $L_{s-1}$ and $R_{s-1}$; an order induced by their labels will make this step deterministic. Let $\bar{a}_{0}<\bar{a}_{1}<\cdots<\bar{a}_{k-1}\left(<\right.$ of $\left.\mathscr{A}_{s-1}\right)$ be the labelled successivities at the completion of this step (note that $\bar{a}_{i}$ may not necessarily be labelled $i$ ).

3. In this step, the heart of the construction, we introduce a successivity $\bar{a}$ labelled $k$. If $f_{s}(0), f_{s}(1), \ldots, f_{s}(k)$ are not all defined, place $\bar{a}$ between $L_{s-1}$ and $R_{s-1}$ as in Step 2. If they are all defined, consider their distribution in $\mathscr{B}_{s}$. Let $n$ of the successivities $\left\{f_{s}(1), \ldots, f_{s}(k)\right\}$ lie to the left of $f_{s}(0)$ and the others to the right. Place $\bar{a}$ immediately to the right of $\bar{a}_{n-1}$ (the subscript $n-1$ is as defined in Step 2). Define $R_{s}$ to include the successivity $\bar{a}$ and all those to its right, and $L_{s}$ to include the others.

4. Define $\mathscr{A}_{s}$ as previously indicated, i.e. add points, one to the extreme right, and one between every unlabelled successivity.

This ends the description of the construction.

$\mathscr{S}(\mathscr{A})$ consists of those successivities whose labels are never removed. The labels $0,1, \ldots, k$ are never removed once $C$ has settled on these numbers. It follows that $\mathscr{S}(\mathscr{A})$ is infinite (and is $\leq_{T} C$ ). Thus, if $\mathscr{S}(\mathscr{B})$ is finite, or empty, then $\mathscr{B} \not \mathscr{A}$. If $\mathscr{S}(\mathscr{B})$ is infinite then $f=\lim _{s} f_{s}$ exists and $\mathscr{S}(\mathscr{B}) \geq_{T} f$. If $f \geq_{T} C$ then $\mathscr{S}(\mathscr{B}) \geq_{T} C$ also. If $f \Varangle_{T} C$ then there must be infinitely many $k$ that are enumerated into $C$ after $f$ has settled on $0,1, \ldots, k$. (We use here the fact that, as the enumeration progresses, $f$, like $C$, settles down on increasingly larger initial segments of $\omega$; i.e. if $f_{s}$ agrees with $f$ on some initial segment of $\omega$, then so does $f_{t}$ for every $t \geq s$.) Equivalently, there must be infinitely many $k$ satisfying property $*$, defined by (i), (ii), and (iii) below:

(i) $k$ is enumerated into $C$ at some stage $t(k)$;

(ii) $C$ does not change (on or) below $k$ after stage $t(k)$;

(iii) $f$ does not change on or below $k$ after stage $t(k)$.

These $k$ will allow us to prove that $\mathscr{B} \not \mathscr{A}$.

The crucial observation is that, for each such $k$, because of (ii) above, the successivities labelled $0,1, \ldots, k$ at stage $t(k)$ never have their labels removed. By the construction, and because of (iii) above, all labelled successivities introduced after this stage are placed between $L_{t(k)}$ and $R_{t(k)}$. It follows that, of the successivities labelled at $0,1, \ldots, k$, those in $L_{t(k)}$ remain in $L$ and those in $R_{t(k)}$ remain in $R$. Since infinitely many $k$ satisfy $*$, this implies 
that every successivity of $\mathscr{A}$ is either in $L$ or in $R$, i.e. every successivity of $\mathscr{A}$ either has only finitely many successivities to its left or only finitely many to its right. Thus if $f(0)$, in $\mathscr{B}$, has infinitely many successivities on both sides, then $\mathscr{B} \not \approx \mathscr{A}$. (In this case $\mathscr{A}$ will have order-type $(\eta+2+\eta) \cdot\left(\omega+\omega^{*}\right)$.) If $f(0)$ has $l$ successivities to its left then, once $f(0)$ and these $l$ successivities have been enumerated into $\mathscr{B}$, for every $k$ satisfying $*, f_{t(k)}(1), \ldots, f_{t(k)}(k)$ will contain precisely $l$ labelled successivities to the left of $f_{t(k)}(0)$ and therefore $L_{t(k)}$ will contain precisely $l$ labelled successivities (see Step 3 of the construction). These $l$ successivities will remain in $L$ but every other successivity will move into $R$, since, at the next stage when a $k$ satisfying $*$ is enumerated into $C$, by (iii), $f$ will once again list precisely $l$ successivities to the left of $f(0)$ in $\mathscr{B}$ and consequently (see Step 3 of the construction), a new labelled successivity, whose label is never removed (property (ii)), is introduced immediately to the right of these leftmost $l$ successivities of $\mathscr{A}$. Thus $\mathscr{A}$ will have only $l$ successivities in $L$ (it will have order-type $\left.(\eta+2+\eta) \cdot\left(l+\omega^{*}\right)\right)$, and therefore no successivity with precisely $l$ successivities to its left, i.e. no successivity corresponding to $f(0)$; so $\mathscr{A} \not \approx \mathscr{B}$. A similar argument proves that if, in $\mathscr{B}, f(0)$ has $r$ successivities to its right, then $R$ will have at least one successivity too few $(\mathscr{A}$ will have order-type $(\eta+2+\eta) \cdot(\omega+r))$, and therefore $\mathscr{B} \not \approx \mathscr{A}$.

We begin by construction of $\mathscr{A}_{e}$, the $\varnothing^{\prime \prime}$-priority argument on $\mathfrak{L}_{e}$, with a list of candidates for the pair of separators $\left(\mathscr{S}_{e}^{\prime}, \mathscr{S}_{e+1}^{\prime}\right)$ in $\mathscr{L}_{e}$; i.e. a list $T$ of pairs $\left(\bar{n}_{1}, \bar{n}_{2}\right)$ of tuples of $\omega$ with $\left|\bar{n}_{1}\right|=e+3$ and $\left|\bar{n}_{2}\right|=e+4$. As noted previously, there is a $\Pi_{2}$ predicate $P_{e}$ with $\left(\bar{n}_{1}, \bar{n}_{2}\right) \in P_{e}$ if and only if $\bar{n}_{1}$ and $\bar{n}_{2}$ are complete blocks of size $e+3$ and $e+4$, respectively, with $\bar{n}_{1}<\bar{n}_{2}$ in $\mathfrak{L}_{e}$. At each stage $s$, we look among the first $s$ members of $T$ for the leftmost pair $\left(\bar{n}_{1}, \bar{n}_{2}\right)$ that appears to be in $P_{e}$ at this stage. If no such pair exists, we perform just Step 4 of the basic module construction (leaving $L$ and $R$ as is). If such a pair $\left(\bar{n}_{1}, \bar{n}_{2}\right)$ does exist, we apply, with some modifications, one step of our basic module to the interval between $\bar{n}_{1}$ and $\bar{n}_{2}$, taking it to be $\mathscr{B}_{e}$. The following are then necessary modifications $\left(\left(\bar{n}_{1}, \bar{n}_{2}\right)\right.$ is the leftmost pair in $T$ that appears to be in $P_{e}$ at this stage, and $k$ is the element just enumerated into $C$ ) :

(a) We need to keep track of the pair of tuples we were working with when a label was introduced. Labels introduced while working with the pair $\left(\bar{n}_{1}, \bar{n}_{2}\right)$ will be of the form $\left({ }_{-}, \bar{n}_{1}, \bar{n}_{2}\right)$. Successivities may have many labels (produced while working with different pairs in $T$ ); we shall arrange that:

- Each successivity will have all its labels of the form $\left(i,,_{-},{ }_{-}\right)$for some particular $i$, and no two successivities will both have labels of the form $\left(i,,_{-},{ }_{-}\right)$(for the same $i$ ).

- If there is a successivity labelled $\left(i, \bar{m}_{1}, \bar{m}_{2}\right)$, then, for each $j<$ $i$, there will be a (different) successivity labelled $\left(j, \bar{m}_{1}, \bar{m}_{2}\right)$; this for each pair $\left(\bar{m}_{1}, \bar{m}_{2}\right)$ in $T$. 
(b) All labels introduced while working with pairs to the left of the "current" pair $\left(\bar{n}_{1}, \bar{n}_{2}\right)$, i.e. of the form $\left({ }_{-}, \bar{m}_{1}, \bar{m}_{2}\right)$ for some $\left(\bar{m}_{1}, \bar{m}_{2}\right)$ to the left of $\left(\bar{n}_{1}, \bar{n}_{2}\right)$ in $T$, must be retained. Suppose there are $l$ successivities with such labels. If $l \geq k$, just perform Step 4 of the basic module construction, retaining all the successivities with labels (of any kind), and no others.

(c) If $l<k$, begin by removing all labels of the form $\left(i,,_{-},\right)$with $i \geq k$. (Because of the way $l$ is defined, and the properties listed in (a) above, none of these labels will have been introduced while working with pairs to the left of $\left(\bar{n}_{1}, \bar{n}_{2}\right)$.) Introduce, if necessary, labels of the form $\left(i, \bar{n}_{1}, \bar{n}_{2}\right)$ with $i<k$, until all labelled successivities also have an appropriate label of this form (appropriate in the sense of the first property mentioned in (a) above). Now perform Steps 2, 3 and 4 of the basic module construction (introducing the label $\left(i, \bar{n}_{1}, \bar{n}_{2}\right)$ where the basic module called for the introduction of the label $i$ ).

It is clear that the properties enumerated in (a) above are preserved through the construction at each stage. Also clear is the fact that, at each stage, either $l \geq k$ and no labels are added or removed, or $l<k$ and precisely $k$ labelled successivities remain after the stage is executed. Each of these $k$ successivities will have a label of the form $\left(i, \bar{n}_{1}, \bar{n}_{2}\right)$ for some $i \leq k$.

If $\mathfrak{L}_{e}$ does have a pair of separators, of size $e+3$ and $e+4$, respectively, the leftmost such pair in $T,\left(\bar{n}_{1}, \bar{n}_{2}\right)$ will appear to be in $P_{e}$ infinitely often. The (finitely many) pairs to the left of $\left(\bar{n}_{1}, \bar{n}_{2}\right)$ will eventually cease appearing to be in $P_{e}$ and the number of labels they produce will, after a stage, always be less than the $k$ enumerated into $C$. Once this has occurred, at ever subsequent stage when $\left(\bar{n}_{1}, \bar{n}_{2}\right)$ appears to be in $P_{e}$ (and there are infinitely many of these), all labelled successivities will have labels of the form $\left(_{-}, \bar{n}_{1}, \bar{n}_{2}\right)$. Labels introduced subsequently while working with pairs to the right of $\left(\bar{n}_{1}, \bar{n}_{2}\right)$ will always be removed, or coupled with a label of the form $\left(_{-}, \bar{n}_{1}, \bar{n}_{2}\right)$ (this at the next stage when $\left(\bar{n}_{1}, \bar{n}_{2}\right)$ appears to be in $\left.P_{e}\right)$. It follows that, after initial faltering steps, and perhaps with irrelevant steps interspersed (the effects of which will always subsequently be nullified), the construction proceeds just as described in the basic module when applied to the linear order $\mathscr{B}_{e}$ lying between $\bar{n}_{1}$ and $\bar{n}_{2}$ in $\mathfrak{L}_{e}$. Consequently the arguments of the basic module apply to show that either $\mathscr{B}_{e} \not \mathscr{A}_{e}$ or $\mathscr{S}\left(\mathscr{B}_{e}\right) \geq_{T} C$ and therefore $S\left(\mathfrak{L}_{e}\right) \geq_{T} C$ (since $\left.S\left(\mathfrak{L}_{e}\right) \geq_{T} S\left(\mathscr{B}_{e}\right)\right)$. That $S\left(\mathscr{A}_{e}\right) \leq_{T} C$ follows from the fact that no labels of the form $\left(i,,_{-},\right)$with $i \leq k$ are ever removed once $C$ has settled on the numbers $\leq k$.

Meshing the construction of the various $\mathscr{A}_{e}$ (using a separate enumeration of $C$ for each $\mathscr{A}_{e}$, ) and placing them between the blocks $\mathscr{S}_{e}$ and $\mathscr{S}_{e+1}$ of size $e+3$ and $e+4$, respectively, produces the recursive linear order $\mathfrak{A}=\mathscr{S}_{0}+\mathscr{A}_{0}+$ $\mathscr{S}_{1}+\mathscr{A}_{1}+\cdots$. If $\mathfrak{L}_{e} \cong \mathfrak{A}$ then $\mathfrak{L}_{e}$ must be of the form $\mathscr{S}_{0}^{\prime}+\mathscr{B}_{0}+\mathscr{S}_{1}^{\prime}+\mathscr{B}_{1}+\cdots$ 
with each $\mathscr{B}_{i} \cong \mathscr{A}_{i}$. But $\mathscr{B}_{e}$ is the interval between the least (and only) pair of separators $\left(\mathscr{S}_{e}^{\prime}, \mathscr{S}_{e+1}^{\prime}\right)$ of size $e+3$ and $e+4$, respectively; consequently, since $\mathscr{B}_{e} \cong \mathscr{A}_{e}$, we have that $S\left(\mathfrak{L}_{e}\right) \geq_{T} C$. That $S(\mathfrak{A}) \equiv_{T} C$ follows from this (which implies that $S(\mathfrak{A}) \geq_{T} C$ ), and the fact that the $S\left(\mathscr{A}_{e}\right)$ are, uniformly in $e, \leq_{T} C$ (which implies that $S(\mathfrak{A}) \leq_{T} C$ ).

The employment of separators in the last proof was suggested to us by Jockusch and Soare [8] where a construction involving separators is used to show that for every r.e., non-recursive set $C$ there is a $\Pi_{1}$ linear order $\mathfrak{A} \equiv_{T} C$ with no recursive copy. We believe they have provided a useful technique for proving results like these, that guarantee the existence of a structure with an intrinsically nonrecursive property. Previous proofs of such results code sets into the classical isomorphism type; see for example Feiner [3] and [4], Rosenstein [12, Theorem 16.30]. $\S 3$ of Remmel [11], $\S 5$ of Goncharov [6] and Theorems 2.1.1, 2.2.3, and 2.3.1 of Moses [9]. We mention these coding arguments again toward the end of $\S 2$.

We note that the $\varnothing^{\prime \prime}$-priority argument we use to construct the $\mathscr{A}_{i}$ is slightly more intricate than that used in Jockusch and Soare [8] because of the coding employed to force $S\left(\mathfrak{L}_{e}\right) \geq_{T} C$; the argument of [8] would guarantee only that $S\left(\mathfrak{L}_{e}\right)$ is nonrecursive. Adding a similar coding argument to the construction in [8] would prove that, for every r.e., nonrecursive set $C$, there is a $\Pi_{1}$ linear order $\mathfrak{A} \equiv_{T} C$ with $\mathfrak{L} \geq_{T} C$ for every $\Pi_{1}$ copy $\mathfrak{L}$ of $\mathfrak{A}$. This cannot be improved to replace $\Pi_{1}$ by $\Delta_{2}$ since the classic result of Richter that no linear ordering has a nonzero degree actually shows that no $\Pi_{1}$ linear ordering has a $\Delta_{2}$ degree.

\section{DiSCRETE LINEAR ORDERS}

Theorem 1 suggests the problem of classifying, by their classical isomorphism type, those recursive linear orders with intrinsically complete successivities. We show in Theorem 2 that no discrete linear order is a member of this class. There is however a recursive discrete linear order with intrinsically non-low successivities but, surprisingly, none with the successivities intrinsically nonsemi-low. We discuss these results after Theorem 2 and end the section with a proof that every $\Pi_{1}$ discrete linear order on a semi-low universe has a recursive copy.

The construction in Theorem 2 is based on a $\Pi_{2}$ construction of a recursive linear order of type $\left(\omega^{*}+\omega\right) \cdot \tau$ from a $\Pi_{2}$ linear oder of type $\tau$. This basic construction appears also in proofs of the main results in Watnick [15] and Downey and Moses [2] where it is modified to prove other results. Consequently we only sketch this basic construction, devoting our proof to a description of the modification necessary to arrange that the recursive linear order constructed has incomplete successivities. We do present all the formal definitions necessary for the basic construction; Downey and Moses [2] offers a rigorous verification of its details. 
Theorem 2. Every recursive discrete linear order has a recursive copy $\mathfrak{A}$ with $S(\mathfrak{A})$ incomplete. In fact, for each r.e., nonrecursive set $C$, there is a recursive copy $\mathfrak{A}$ with $S(\mathfrak{A}) \Varangle_{T} C$.

Proof. Let $\mathfrak{L}$ be a recursive discrete linear order, $C$ an r.e., nonrecursive set and $\tau$ the order-type of some $\Pi_{2}$ choice set $B$ of $\mathfrak{L}$. We construct a recursive linear order $\mathfrak{A}$ of type $\left(\omega^{*}+\omega\right) \cdot \tau$ (so that $\mathfrak{A} \cong \mathfrak{L}$ ), and we meet the set of requirements $R_{e}: \Phi_{e}(S(\mathfrak{A})) \neq C$, where $\Phi_{e}(S(\mathfrak{A}))$ is the eth oracle machine with oracle $S(\mathfrak{A})$. The choice set $B$ will govern our construction of $\mathfrak{A} ;|\mathfrak{A}|$ will be an r.e. subset of $\omega$. Since $B$ is $\pi_{2}$, there is a recursive binary relation $D$ satisfying $n \in B \Leftrightarrow \exists^{\infty} x D(n, x)$. A result in Jockusch [7] allows us to assume that $D$ has the additional property:

$$
\forall n \in B \exists^{\infty} x \forall k \leq n \quad[k \in B \Rightarrow D(k, x)] .
$$

We offer a brief verification of this fact: Given a recursive $D_{1}$ satisfying the weaker property, we produce a recursive $D_{2}$ satisfying, in addition, property $(*)$. For $S$ a finite subset of $\omega$, we define the phrase $S$ is alive at stage $s$ as follows. $S$ is not alive at any stage prior to the stage $\max S$. For $s>\max S$, we say $S$ is alive at stage $s$ if, writing $t$ for the last stage prior to $s$ when $S$ was alive, or $t=\max S$ if $S$ has never before been alive, we have $\forall n \in$ $S \exists x\left((t<x \leq s) \wedge D_{1}(n, x)\right)$. We then define $D_{2}$ by: $(n, x) \in D_{2}$ if and only if there is a subset $S \subseteq\{1, \ldots, x\}$, with $n \in S$, and with $S$ alive at stage $x$. It is not difficult to verify that $D_{2}$ has the required properties.

Our strategy for building an interval $I(i)$ of order-type $\omega^{*}+\omega$ for each $i \in B$ is to use $\Pi_{2}$ guessing, with the aid of the usual $2^{<\omega}$ tree. Each node $\sigma$ with $|\sigma|=i$ will correspond to a guess as to which of the numbers $j \leq i$ are in $B$. Property $(*)$ will ensure that the nodes corresponding to correct guesses will govern our construction at infinitely many stages. We define intervals $I(\sigma)$ for each node $\sigma$ and write $I(\sigma, s)$ for the version defined at stage $s$. If $i \in B$ and $\sigma$ is the unique node of length $i$ that corresponds to a correct guess, then $I(\sigma)$ will be an interval of type $\omega^{*}+\omega$. Every other $I(\sigma)$ will be finite (or empty) and will become part of some $I(\sigma)$ of type $\omega^{*}+\omega$. For a more leisurely account of this basic construction see Theorem 1 of Downey and Moses [2].

Our strategy for meeting the requirements $R_{e}$ is to preserve agreements between $\Phi_{e, s}\left(S\left(\mathfrak{A}_{s}\right)\right)$ and $C_{s}$ and argue from this that if $\Phi_{e}(S(\mathfrak{A}))=C$ then $C$ must be recursive. To preserve the agreement $\Phi_{e, s}\left(S\left(\mathfrak{A}_{s}\right) ; x\right) \downarrow=C_{s}(x)$ we must arrange that the initial segment $S(e)$ of $S\left(\mathfrak{A}_{s}\right)$ which is used in the computation of $\Phi_{e, s}\left(S\left(\mathfrak{A}_{s}\right) ; x\right)$ remains an initial segment of $S(\mathfrak{A})$. Since $S(\mathfrak{A})$ is determined by our block-building strategy, we consider, instead of sets $S(e)$ for $\Phi_{e}$, sets $S(\sigma)$ for each node $\sigma$. The sets $S(\sigma)$ with $\sigma$ of length $e$ will be versions of $S(e)$; and the $S(\sigma)$ with $\sigma$ the unique node of length $e$ corresponding to a correct guess (with regard to $B$ ) will witness our meeting $R(e)$. We write $S(\sigma, s)$ for the version of $S(\sigma)$ defined at stage $s ; S(\sigma, s)$ will be an initial segment of $S\left(\mathfrak{A}_{s}\right)$. At the stage $s$ that we decide to preserve the agreement $\Phi_{|\sigma|, s}(S(\sigma, s) ; x) \downarrow=C(x)$ we will make $S(\sigma, s)$ an initial segment of 
$S\left(\mathfrak{A}_{s}\right)$; i.e., all $i \leq \max S(\sigma, s)$ with $i \notin S(\sigma, s)$ will have $\bar{s}_{i}$ a nonsuccessivity in $\mathfrak{A}_{s}$. All that remains in order to preserve this agreement is to ensure that $S(\sigma, s) \subseteq S(\mathfrak{A})$. We will be able to guarantee this under the assumption that we never again act for a node $\alpha<\sigma$. If $\sigma$ corresponds to a correct guess (with regard to $B$ ) there will be a stage when this assumption is in fact true. In this manner we arrange that our construction along the "true path" is a finite injury priority construction of a recursive linear order $\mathfrak{A} \cong \mathfrak{L}$ and that we meet all the requirements $R_{e}$.

We present the formal definitions:

The terminology relating to the $2^{<\omega}$ tree ( $\alpha$ is above $\beta, \alpha$ is to the left of $\beta, \alpha \subset \beta$ and $\alpha<\beta)$ is standard; see, for example, Soare [14] or Downey and Moses [2]. For each $i \leq|\sigma|, \sigma$ guesses that $i \in B$ if $\sigma(i)=0$ and that $i \notin B$ if $\sigma(i)=1$ and $\sigma$ 's guess seems correct at stage $s$ if and only if

$$
\forall i \leq|\sigma| \quad[(i, s) \in D \Leftrightarrow \sigma \text { guesses that } i \in B] .
$$

Notice that if $\sigma$ 's guess seems correct at stage $s$ then this is also true of every $\alpha \subset \sigma$ and of exactly one of $\sigma^{\wedge} 0, \sigma^{\wedge} 1$. If $\sigma$ 's guess is correct it will seem so at infinitely many stages, this because of property $(*) . P_{s}$ is the unique path consisting of those nodes whose guesses seem correct at stage $s . P$ is the true path, consisting of those nodes whose guesses are correct; i.e. those $\sigma$ satisfying $\sigma(i)=0 \Leftrightarrow i \in B$, for each $i \leq|\sigma|$. We write $\left.P_{s}\right|_{n}$ and $\left.P\right|_{n}$ for the restrictions of these paths to nodes of length $\leq n$. Notice that, uniformly in $s$, the $P_{s}$ are recursive (since $D$ is) and that $P$ is $\Pi_{2}$ (since $B$ is). Because of property $(*)$, each $\sigma \in P$ will appear on $P_{s}$ at infinitely many stages $s$.

The requirement $R_{|\sigma|}$ will require attention at stage $s$ if $\left.\sigma \in P_{s}\right|_{s}$ and if it is possible, at this stage, to extend the agreement between $\Phi_{|\sigma|, s}$ and $C_{s}$. Precisely, $R_{e}$ requires attention at stage $s$ if there is node $\left.\sigma \in P_{s}\right|_{s}$ with $E=|\sigma|$, a set $E$ and an element $x$ satisfying the following three clauses:

1. $\Phi_{c, s}(E ; x) \downarrow=C_{s}(x)$.

2. $x=\max \left\{y: \Phi_{e, s}(S(\sigma, s-1) ; y) \downarrow=C_{s}(y)\right\}+1$.

3. $E$ satisfies the following:

(a) $E \subseteq S\left(\mathfrak{A}_{s-1}\right)$;

(b) $\left\{i: \bar{s}_{i} \subseteq I(\alpha, s-1)\right.$ for some $\left.\alpha \leq \sigma\right\} \subseteq E$;

(c) $\bigcup\{S(\alpha, s-1): \alpha \leq \sigma\} \subseteq E$; and

(d) $\forall \alpha \subseteq \sigma, \forall i \in E$, either $\bar{s}_{i} \subseteq I(\alpha, s-1)$ or $\bar{s}_{i} \cap I(\alpha, s-1)=\varnothing$.

Our reasons for the requirements (a)-(d) are as follows. We intend to make $S(\sigma, s)=E$, hence requirement (a). In addition, to ensure that $S(\sigma, s)$ is an initial segment of $S(\mathfrak{A})$, we intend to make it an initial segment of $S\left(\mathfrak{A}_{s}\right)$ itself, by introducing points between the elements of some $\bar{s}_{i}$ if necessary. Requirement (b) ensures that while doing so we do not introduce points within and $I(\alpha, s-1)$ with $\alpha \leq \sigma$. Consequently, we will extend such intervals $I(\alpha, s-1)$ at the ends only and, if $\alpha$ correctly guesses that $|\alpha|=B$, will produce an interval $I(\alpha)$ of order-type $\omega^{*}+\omega$. Requirement (c) similarly ensures that no $\bar{s}_{i}$ with $i \in S(\alpha, s-1)$ for $\alpha \leq \sigma$ is turned into a nonsuccessivity at this 
stage. Requirement (d) is directed toward ensuring that $S(\sigma, s) \subseteq S(\mathfrak{A})$. Our intention is to leave $\mathfrak{A}_{s}$ as a union of disjoint intervals $I(\alpha, s)$ with $\alpha \subseteq \sigma$. The intervals of $\mathfrak{A}_{s-1}$ not contained in any $I(\alpha, s-1)$ with $\alpha \subseteq \sigma$ will be absorbed, wholly, into some $I(\alpha, s)$ with $\alpha \subseteq \sigma$. Requirement (d) allows us to be quite casual about this absorption without running the risk that an $\bar{s}_{i}$ with $i \in S(\sigma, s)$ becomes split between separate intervals $I(\alpha, s)$ and $I(\beta, s)$ with $\alpha, \beta \subseteq \sigma$. If we never again act for a node $\leq \sigma$, these intervals $I(\alpha, s)$ with $\alpha \subseteq \sigma$ will never have points introduced internally, i.e. they will be extended at the ends only; consequently $S(\sigma, s)$ will be a subset of $S(\mathfrak{A})$.

The construction at stage $s$ may call for the introduction of a new element into the linear order. By this is meant the least element of $\omega$ not yet in $\mathfrak{A}$ and not a member of any $\bar{s}_{i}$ with $i \leq \max S(\sigma, s-1)$ for any one of the (finitely many) sets $S(\sigma, s-1)$. This is so as not to jeopardize our meeting of $R_{|\sigma|}$ by inadvertently introducing a successivity $\bar{s}_{i}$ with $i \leq \max S(\sigma, s-1)$.

\section{The construction at stage $s$.}

Look for the least $e$ with $R_{e}$ requiring attention at this stage. If there is one, let $E$ and $\sigma$ be as in the definition of " $R_{e}$ requiers attention at stage $s$ ". Define $S(\sigma, s)=E$ and introduce a new element between the points of each $\bar{s}_{i}$ with $i \in S\left(\mathfrak{A}_{s-1}\right)$ and $i \leq \max E$ but $i \notin E$. If no $R_{e}$ requires attention, let $\sigma$ be the node on $P_{s}$ with $|\sigma|=s$; define $S(\sigma, s)=S(\sigma, s-1)$ and read $s$ for $e$ in what follows. The rest of the construction proceeds without regard to whether or not there is an $R_{e}$ requiring attention at this stage. Define:

$$
\begin{aligned}
& S(\alpha, s)=S(\alpha, s-1) \text { for every } \alpha<\sigma, \\
& I(\alpha, s)=I(\alpha, s-1) \text { for every } \alpha \text { to the left of } P_{s} \text { and } \\
& S(\alpha, s)=I(\alpha, s)=\varnothing \text { for every } \alpha>\sigma .
\end{aligned}
$$

Then modify $\mathfrak{A}_{s-1}$ using $\left.P_{s}\right|_{e}$ as described below; we will in the process define $I(|\alpha|, s)$ for $\alpha \subseteq \sigma$. We use the nodes of $\left.P_{s}\right|_{e}$ in turn, beginning with the root $\lambda$. For $\lambda$, leave $\mathfrak{A}_{s-1}$ as is. After modifying $\mathfrak{A}_{s-1}$ for a node on $\left.P_{s}\right|_{e}$ consider its successor $\sigma$ on $\left.P_{s}\right|_{e}$.

Case 1. $\sigma$ guesses that $|\sigma| \notin B$.

Define $I(\sigma, s)=I(\sigma, s-1)(=\varnothing)$. Leave everything else as is.

Case 2. $\sigma$ guesses that $|\sigma| \in B$ and $I(\sigma, s-1)=\varnothing$.

The construction will guarantee that the intervals in $\{I(\alpha, s): \alpha \subseteq \sigma\}$ either are all empty or form a partition of the existing linear order. If they are all empty, define $I(\sigma, s)$ to be all of the existing linear order. Otherwise define $i(\sigma, s)=\{a\}$ where $a$ is a new element, and place it among the intervals in $\{I(\alpha, s): \alpha \subset \sigma\}$ as dictated by $\mathfrak{L}$. That is, place $I(\sigma, s)$ to the left of such an $I(\alpha, s)$ if and only if $|\sigma|<|\alpha|$ in $\mathfrak{L}$. Leave all else as is.

Case 3. $\sigma$ guesses that $|\sigma| \in B$ and $I(\sigma, s-1) \neq \varnothing$.

Let $D_{1}$ and $D_{2}$ be the intervals on either side of $I(\sigma, s-1)$ and (strictly) between it and all other intervals $i(\alpha, s-1)$ or $i(\alpha, s)$ with $\left.\alpha \in P_{s}\right|_{e}$. Define $I(\sigma, s)=D_{1} \cup I(\sigma, s-1) \cup D_{2}$. Leave all else as is. 
This ends the modification for $\sigma$. After modifying for each node on $\left.P_{s}\right|_{e}$ add a new element to each end of each interval $I(\sigma, s)$ with $\left.\sigma \in P_{s}\right|_{e}$ and guessing that $|\sigma| \in B$. This ends the construction at stage $s$.

The construction is effective and produces a recursive linear order $\mathfrak{A}$. The only nodes $\alpha$ that may have $I(\alpha, s) \neq \varnothing$ are those that end in 0 (see Case $1)$. If $I(\sigma, s) \neq \varnothing$ then every $\alpha \subseteq \sigma$ that ends in 0 also has $I(\alpha, s) \neq \varnothing$. It follows, by Cases 2 and 3, that the intervals in $\left\{I(\sigma, s): \sigma \in P_{s}\right\}$ either are all empty or they partition $\mathfrak{A}_{s}$ and, by Case 2, are ordered as dictated by $\mathfrak{L}$. A crucial fact is that for every $\sigma$ and $s, S(\sigma, s)$ is an initial segment of $S(\mathfrak{A})$. That this is true at each stage when $S(\sigma, s)$ is extended follows from $3(\mathrm{a})$ and 3(b) in the definition of " $R_{e}$ requires attention" (these guarantee that $\left.S(\sigma, s) \subseteq S\left(\mathfrak{A}_{s}\right)\right)$ and the fact that if $i \leq \max S(\sigma, s)$ but $i \notin S(\sigma, s)$ then either $\bar{s}_{i}$ is a non-successivity in $\mathfrak{A}_{s}$ or at least one element of $\bar{s}_{i}$ will not enter $\mathfrak{A}$ as long as $S(\sigma, s)$ is defined (see the definition of "new element"). At subsequent stages $t$, either $S(\sigma, s)$ will be reduced to $\varnothing$, or, because of $3(\mathrm{~b})$ and $3(\mathrm{c})$ and the fact that we extend intervals at the ends only, will remain an initial segment of $S(\mathfrak{A})$. That $\mathfrak{A}$ has order-type $\left(\omega^{*}+\omega\right) \cdot \tau$ and that each $R_{e}$ is met follow from:

Lemma. For each $\sigma \in P$ there are only finitely many stages $s$ with $\sigma \in P_{s}$ at which $R_{|\sigma|}$ requires attention.

Proof. Let $\sigma$ be the counterexample with $|\sigma|$ the least. Let $s_{0}$ be a stage after which no $\alpha<\sigma$ is ever the bottom-most node of $\left.P_{s}\right|_{e}$ (as used in the construction). Such a stage exists, by the inductive hypothesis if $\alpha \in P$, and, if $\alpha$ is to the left of $P$, by the fact that $\alpha$ 's guess eventually stops seeming correct. It follows that

$$
S\left(\sigma, s_{0}\right) \subseteq S\left(\sigma, s_{0}+1\right) \subseteq S\left(\sigma, s_{0}+2\right) \subseteq \cdots .
$$

Let $t(0)<t(1)<t(2)<\cdots$ be a sequence of stages after $s_{0}$ with $\sigma \in P_{t(i)}$ and $R_{|\sigma|}$ requiring attention at stage $t(i)$ for each $i$. We will act for $R_{|\sigma|}$ at these stages and each $S(\sigma, t(i))$ will be an initial segment of $S(\mathfrak{A})$. It follows that, for each $x \in \omega, \Phi_{|\sigma|, t(x)}(S(\sigma, t(x)) ; x)=C_{t(x)}(x)$ and that $C_{t(x)}(x)=C(x)$; otherwise a disagreement between $\Phi_{|\sigma|}$ and $C$ would occur and be preserved, and $R_{|\sigma|}$ would not be a counterexample to the lemma. This implies that $C$ is recursive, which it is not; hence the lemma is proved.

We can now prove that for each $\sigma \in P$ there is a stage after which $I(\sigma, s)$ is never destroyed and that every element of $|\mathfrak{A}|$ eventually enters some such $I(\sigma, s)$. These together imply that $\mathfrak{A}$ has type $\left(\omega^{*}+\omega\right) \cdot \tau$. That each $R_{e}$ is met follows from the fact that $C$ is nonrecursive, arguing just as in the lemma.

We note that, in contrast to the last result,

There is a recursive discrete linear order $\mathfrak{A}$ with $S(\mathfrak{A})$ intrinsically non-low, i.e. no recursive $\mathfrak{L} \cong \mathfrak{A}$ has $S(\mathfrak{L})$ low. 
A set $S \leq_{T} \varnothing^{\prime}$ is low if $S^{\prime} \equiv_{T} \varnothing^{\prime}$, equivalently, if $\Sigma_{1}^{S} \subseteq \Pi_{2}$. To construct $\mathfrak{A}$ we begin with a $\Pi_{2}$ linear order, of type $\tau$ say, that has no $\Sigma_{2}$ copy (equivalently, no $\Pi_{1}$ copy); such an order exists by Rosenstein [12; see Proposition 16.33 and Corollary 16.35]. By Watnick [15] there is a recursive linear order of type $\left(\omega^{*}+\omega\right) \cdot \tau$; let $\mathfrak{A}$ be one such linear oder. No recursive copy $\mathfrak{L}$ of $\mathfrak{A}$ has $S(\mathfrak{L})$ low since $B(x, y)$, which is r.e. in $S(\mathfrak{L})$, would be $\Pi_{2}$ in this case. Consequently, the subset $B$ of $|\mathfrak{L}|$ defined by $b \in B \Leftrightarrow \forall x<b \neg B(x, b)$ $(<$ in $\omega)$ would be a $\Sigma_{2}$ linear order of type $\tau$; there are none of these by our choice of $\tau$.

This last result is tight in the sense that it fails if we replace "low" with "semilow". A co-r.e. set is semi-low if $\left\{e: W_{e} \cap S \neq \varnothing\right\} \leq_{T} \varnothing^{\prime}$ (whereas it is low if $\left\{e: \exists u \in W_{e}\right.$ with $\left.\left.D_{u} \subseteq S\right\} \leq_{T} \varnothing^{\prime}\right)$.

Every recursive discrete linear order $\mathfrak{L}$ has a recursive copy $\mathfrak{A}$ with $S(\mathfrak{A})$ semi-low.

We present a brief description of the $\varnothing^{\prime}$-priority construction of $\mathfrak{A}$. Writing $\mathfrak{L}_{s}$ for the restriction of $\mathfrak{L}$ to $\{0,1, \ldots, s\}$ we define $\mathfrak{A}_{s}$ on the same universe and $g_{s}: \mathfrak{A}_{s} \cong \mathfrak{L}_{s}$. We will attempt to meet the requirements $R_{e}: W_{e} \cap S(\mathfrak{A}) \neq$ $\varnothing$. At each stage $s$ we define $w(e, s)$ to be the least (in $\omega$ ) element of $W_{e, s} \cap S\left(\mathfrak{A}_{s}\right)$ if it exists. We aim to make $w(e)=\lim _{s} w(e, s)$ exist and witness our meeting $R_{e}$. We will fail in this aim only if $W_{e} \cap S(\mathfrak{A})=\varnothing$. Our actions to meet $R_{e}$ at stage $s+1$ should leave $g_{s+1}=g_{s}$ on the first $e$ members of $\left[\mathfrak{A}_{s}\right]$ and $\left|\mathfrak{L}_{s}\right|$ and on the members of $\bar{s}_{w\left(e^{\prime}, s\right)}$ for every $e^{\prime}<e$ with $w\left(e^{\prime}, s\right)$ defined. Consequently, we define the set of "fixed points" for $R_{e}$ at stage $s$ to be

$$
D(e, s)=\{0,1 \ldots, e\} \cup\left\{g_{s}^{-1}(0), g_{s}^{-1}(1), \ldots, g_{s}^{-1}(e)\right\} \cup\left\{\bar{s}_{w\left(e^{\prime}, s\right)}: e^{\prime}<e\right\} .
$$

$R_{e}$ requires attention at stage $s+1$ if the new element $s+1$ of $\mathfrak{L}$ lies between the points of $g_{s}\left(\bar{s}_{w(e, s)}\right) . R_{e}$ receives attention at stage $s+1$ only if neither of the elements of $\bar{s}_{w(e, s)}$ is in $D(e, s)$. If this occurs we act to retain $w(e, s)$ in $S\left(\mathfrak{A}_{s+1}\right)$ as follows. If $\bar{s}_{w(e, s)}=[a, b]$, define $g_{s+1}(b)=s+1$, the new point of $\mathfrak{L}$, and define $g_{s+1}(s+1)=g_{s}(b)$, i.e. introduce $s+1$ in $\mathfrak{A}$ immediately to the right of $b$; let $g_{s+1}=g_{s}$ on all other points. This moving of $g_{s}(b)$ towards $g_{s}(a)$ need occur only finitely often since the successor of $g_{s}(a)$ must eventually be enumerated into $\mathfrak{L}$. (Note that the same element of $\bar{s}_{w(e, s)}$ must remain fixed through all such actions; we choose to fix the left element $a$.) From this fact we can show that each $R_{e}$ receives attention at most finitely often and that consequently a recursive linear order $\mathfrak{A}$ and an isomorphism $g: \mathfrak{A} \cong \mathfrak{L}$ are defined. To see that $S(\mathfrak{A})$ is semi-low consider the recursive function

$$
f(e, s)= \begin{cases}0 & \text { if } w(e, s) \text { exists } \\ 1 & \text { otherwise. }\end{cases}
$$

For any $e$, let $s \geq e$ be a stage after which no $R_{e^{\prime}}$ with $e^{\prime}<e$ ever receives attention (and therefore $D(s, e)=D(e))$ and the predecessors and successors of the elements of $g_{s}(D(e))$ have all been enumerated into $\mathfrak{L}$. If, at some stage 
$t \geq s, w(e, t)$ is defined, then $f(e)=0$; otherwise $f(e)=1$. The choice of $w(e, t)$ may change after $t$, finitely often, if smaller elements are enumerated into $W_{e} \cap S(\mathfrak{A})$; but this will settle on some $w(e)$ which our actions to meet $R_{e}$ will retain as a member of $W_{e} \cap S(\mathfrak{A})$. It follows that $f(e)=\lim _{s} f(e, s)$ exists and is the characteristic function of $\left\{e: W_{e} \cap S(\mathfrak{A}) \neq \varnothing\right\}$. By the Limit Lemma, this set is $\leq_{T} \varnothing^{\prime}$ and so $S(\mathfrak{A})$ is semi-low.

This last argument works also for recursive linear orders of type $\omega \cdot \tau$ and a similar argument, keeping $g_{s}(b)$ fixed and moving $g_{s}(a)$ toward it, works for the order-types $\omega^{*} \cdot \tau$. Such arguments will fail for linear orders that contain infinitely many blocks of finite length. There are in fact such recursive linear orders $\mathfrak{A}$ with $S(\mathfrak{A})$ intrinsically non-semi-low-produced by the coding arguments that construct a recursive linear order $\mathfrak{A}$ with $S(\mathfrak{A})$ intrinsically nonrecursive. Such an $\mathfrak{A}$ may be produced by coding a $\Sigma_{3}$-complete set into the set $B=\{n: \mathfrak{A}$ has a complete block of size $n\}$. It is clear that $S(\mathfrak{A})$ being recursive would make $B \quad \Sigma_{2}$ and, since $B$ is determined by the classical isomorphism type of $\mathfrak{A}$, it follows that $S(\mathfrak{A})$ is intrinsically nonrecursive. Such arguments originated in Feiner [3] and [4]; for various extensions see Remmel [11], Goncharov [6], Rosenstein [12, Theorem 16.30] and Moses [9; see the end of $\S 1$ for theorem/section numbers]. To see that the $S(\mathfrak{A})$ so constructed is also intrinsically non-semi-low we use the fact that if it is semi-low then the relations $I(x)$ and $F(x)$ defined by $\forall y<x \neg S(y, x)$ and $\forall y>x \neg S(x, y)$ respectively $(<$ of $\mathfrak{A})$ are both $\Sigma_{2}$ in $\mathfrak{A}$. We show this for $I(x)$ :

For each $a \in|\mathfrak{A}|$ define $W_{\langle a\rangle}$ to be the r.e. set $\{(x, a): x<a$ in $\mathfrak{A}\}$. Then $a \in I$ if and only if $W_{\langle a\rangle} \cap S(\mathfrak{A})=\varnothing$. If $S(\mathfrak{A})$ is semi-low there is a recursive function $f$ with $W_{\langle a\rangle} \cap S(\mathfrak{A})=\varnothing$ if and only if $W_{f(\langle a\rangle)}$ is finite; this is a $\Sigma_{2}$ predicate. Since $[a, b]$ is a complete block in $\mathfrak{A}$ if and only if $I(a) \wedge F(b) \wedge B(a, b)$, it follows that the set $B$ defined above is $\Sigma_{2}$. Since $B$ is determined by the classical isomorphism type, we have that the recursive linear order $\mathfrak{A}$ so constructed has $S(\mathfrak{A})$ intrinsically non-semi-low.

We briefly consider a related issue, concerning $\Pi_{1}$ linear orders. It is known (see Roy and Watnick [13]) that there is a $\Pi_{1}$ discrete linear order with no recursive copy. However, every low $\Pi_{1}$ discrete linear order does have a recursive copy. We show that, in fact,

Every semi-low $\Pi_{1}$ discrete linear order has a recursive copy.

Let $\mathfrak{A}$ be a semi-low $\Pi_{1}$ discrete linear order, defined as a subset $A$ of $Q$. Consider $B \subseteq A$ defined by

$$
b \in B \Leftrightarrow \forall x<b \quad(x \notin A \text { or } \forall y \exists z>y \text { (with } z \in A \text { and }
$$
$z$ lying between $x$ and $b$ in $\mathfrak{A})$ ).

(Here < is that of $\omega$.) Then $B$ is a choice set for $\mathfrak{A}$. We show that $B$ is $\Pi_{2}$ and therefore, by Watnick [15], that $\mathfrak{A}$ has a recursive copy. For each triple $x$, $b, y$ define $W_{\langle x, b, y\rangle}$ to be the r.e. set consisting of those $z>y$ (in $\omega$ ) that 
lie between $x$ and $b$ in $Q$. Then

$$
b \in B \Leftrightarrow \forall x<b \quad\left(x \notin A \text { or } \forall y\left(W_{\langle x, b, y\rangle} \cap A \neq \varnothing\right)\right) .
$$

Since $A$ is semi-low, there is a recursive function $f$ such that $W_{\langle x, b, y\rangle} \cap A \neq \varnothing$ if and only if $W_{f(\langle x, b, y\rangle)}$ is infinite. Consequently

$$
b \in B \Leftrightarrow \forall x<b \quad\left(x \notin A \text { or } \forall y\left(W_{f(\langle x, b, y)} \text { is infinite }\right)\right) .
$$

The relation $x \notin A$ is $\Sigma_{1}$ and the relation between the brackets is $\Pi_{2}$. It follows that $B$ is a $\Pi_{2}$ set and therefore, by Watnick [15], that $\mathfrak{A}$ has a recursive copy.

\section{INTRINSICALLY $w t t$-COMPLETE SUCCESSIVITIES}

We show that, in contrast with Theorem 1, the class of recursive linear orders with intrinsically $w t t$-complete successivities is empty.

Theorem 3. Every recursive linear order has a recursive copy $\mathfrak{A}$ with $S(\mathfrak{A})$ wttincomplete. In fact, for each r.e., nonrecursive set $C$, there is a recursive copy $\mathfrak{A}$ with $S(\mathfrak{A}) \Varangle_{\text {wit }} C$.

Proof. Let $\mathfrak{L}$ be a recursive linear order and $C$ an r.e., nonrecursive set. We use a $\varnothing^{\prime}$-priority argument to construct a recursive copy $\mathfrak{A}$ of $\mathfrak{L}$ meeting the requirements:

$$
R(e, i): \Phi_{e}(S(\mathfrak{A})) \neq C \text { or } \phi_{i} \text { does not majorize } u(S(\mathfrak{A}), e) .
$$

$\Phi_{e}(S(\mathfrak{A}))$ is the $e$ th oracle machine with oracel $S(\mathfrak{A}), \phi_{i}$ the $i$ th partial recursive function and $u(S(\mathfrak{A}), e)$ the use function for the computation $\Phi_{e}(S(\mathfrak{A}))$. The requirements $R(e, i)$ have a priority ordering determined by $\omega^{2}$. At each stage $s$ we define $\mathfrak{A}_{s}$ on $\{0,1, \ldots, s\}$ and an isomorphism $g_{s}$ from $\mathfrak{A}_{s}$ to $\mathfrak{L}_{s}$, the restriction of $\mathfrak{L}$ to $\{0,1, \ldots, s\}$. We arrange that $g$ eventually settles on each element of $|\mathfrak{A}|$ and $|\mathfrak{L}|$. With this in mind we define for each $R(e, i)$ and $s$ the set

$$
D(e, i, s)=\left\{g_{s}(j): j \leq\langle e, i\rangle\right\} \cup\{j: j \leq\langle e, i\rangle\} .
$$

$\langle e, i\rangle$ is the index of $(e, i)$ in $\omega^{2} . D(e, i, s)$ is the subset of $|\mathfrak{L}|$ on which $g_{s}$ must remain unchanged by any action to meet $R(e, i)$ at stage $s+1$. Arranging that we act only finitely often to meet each $R(e, i)$ will guarantee that $g=$ $\lim _{s} g_{s}$ exists.

Our strategy to meet $R(e, i)$ is to attempt to arrange a disagreement $\Phi_{e}(S(\mathfrak{A}) ; k) \neq C(k)$, working successively with $k=0,1,2, \ldots$ It at some stage $s, \Phi_{e, s}\left(S\left(\mathfrak{A}_{s}\right) ; k\right) \downarrow=C_{s}(k)$ and $\phi_{i, s}(k) \downarrow \geq u\left(S\left(\mathfrak{A}_{s}\right) ; e, k, s\right)$, we try to remove all $j \leq \phi_{i}(k)$ from $S(\mathfrak{A})$. If we succeed in this task by stage $t$ we will have $S\left(\mathfrak{A}_{t}\right) \cap\left\{j: j \leq \phi_{i}(k)\right\}=\varnothing$. Consequently, if $\phi_{i}$ is to majorize $u(S(\mathfrak{A}), e)$, we must have $\Phi_{e}(S(\mathfrak{A}) ; k)=\Phi_{e}(\varnothing, k)$. If we succeed in this strategy for each $k$ without producing a disagreement $\Phi_{e}(S(\mathfrak{A}) ; k) \neq C(k)$, then $C$ must be recursive, which it is not. 
The set $D(e, i, s)$ may not allow us to remove all $j \leq \phi_{i}(k)$ from $S(\mathfrak{A})$. However, there will be a finite set $I$ such that for every $k$ we will be able to remove from $S(\mathfrak{A})$ all $j \leq \phi_{i}(k)$ that are not in $I$. The previous argument is still valid since having $\Phi_{e}(S(\mathfrak{A}))=\Phi_{e}(I)$ would (since $I$ is finite) still imply that $C$ is recursive.

The standard machinery of a $\varnothing^{\prime}$-priority construction provides the bookkeeping; we leave the details to the reader.

If, in the last theorem, the given linear order $\mathfrak{L}$ has $S(\mathfrak{L})$ infinite and if $C$ is incomplete, we can arrange also that $S(\mathfrak{A}) \not C C$ and therefore that $S(\mathfrak{A})$ and $C$ are $w t t$-incomparable. To do this we meet the additional set of requirements $P_{e}: \Phi_{e}(C) \neq S(\mathfrak{A})$. Once again our basic strategy is to attempt to remove elements $j$ from $S(\mathfrak{A})$ but now we do this only if $K$ has changed below $j$ since the stage when $\Phi_{e}(C ; j)$ took on its present value. Either we are successful in preserving one such disagreement between $\Phi_{e}(C)$ and $S(\mathfrak{A})$, or for all but finitely many $j$ (for all $j \notin I$ ), $\Phi_{e}(C)$ changes below $j$ after $K$ does. Consequently, if $P_{e}$ requires attention infinitely often and/or $\Phi_{e}(C)=S(\mathfrak{A})$ we can prove that $C \geq_{T} K$, which it is not.

Theorem 3 , in the case when $S(\mathfrak{L})$ is infinite, may alternatively be proved using the basic strategy of removing elements from $S(\mathfrak{A})$ to construct a recursive copy $\mathfrak{A}$ with $S(\mathfrak{A})$ hyperimmune. (The construction is similar to that employed by Remmel in Theorem 2.8 of [10] to show that every recursive Boolean algebra with infinitely many atoms has a recursive copy in which the set of atoms is hyperimmune.) It follows from a result in Friedberg and Rogers [5, p. 124] that $S(\mathfrak{A})$ is $w t t$-incomplete; in fact, by Downey and Jockusch [1, Theorem 4.12], it is not $w t t$-cuppable.

\section{CONCLUSION}

The problem of classifying those recursive linear orders with intrinsically complete successivities will, we think, prove difficult. Theorem 2 solves the problem for the class of discrete linear orders. We suggest a further test case which exemplifies the problems we encountered in attempting to extend our methods to a general solution: The class of order-types with the property that between every pair of successivities there lie only finitely many other successivities. Are there any such recursive linear orders with intrinsically complete successivities? It is possible to show that, in contrast with discrete order-types, there are such recursive linear orders with intrinsically non-semi-low successivities.

\section{REFERENCES}

1. R. Downey and C. Jockusch, $T$-degrees, jump classes and strong reducibilities, Trans. Amer. Math. Soc. 301 (1987), 103-137.

2. R. Downey and M. Moses, On choice-sets and strongly non-trivial self-embeddings of recursive linear orders, Z. Math. Logik. Grundlagen Math. 35 (1989), 237-246. 
3. L. Feiner, Hierarchies of Boolean algebras, J. Symbolic Logic 35 (1970), 365-373.

4. __ The strong homogeneity conjecture, J. Symbolic Logic 35 (1970), 375-377.

5. R. Friedberg and H. Rogers, Reducibility and completeness for sets of integers, Z. Math. Logik. Grundlagen Math. 5 (1959), 117-125.

6. S. Goncharov, Some properties of the constructivization of Boolean algebras, Sibirsk. Math. Zh. 16 (1975), 203-214.

7. C. Jockusch, Reducibilities in recursive function theory, Ph. D. Thesis, MIT, 1966.

8. C. Jockusch and R. Soare, Degrees of orderings not isomorphic to recursive linear orderings, Ann. Pure Appl. Logic (to appear).

9. M. Moses, Recursive properties of isomorphism types, $\mathrm{Ph}$. D. Thesis, Monash University, 1983.

10. J. Remmel, Recursive isomorphism types of recursive Boolean algebras, J. Symbolic Logic 46 (1981), 572-593.

11. __ Recursive Boolean algebras with recursive sets of atoms, J. Symbolic Logic 46 (1981), 596-616.

12. J. Rosenstein, Linear orderings, Academic Press, 1982.

13. D. Roy and R. Watnick, Finite condensations of recursive linear orders, Ann. Pure Appl. Logic (to appear).

14. R. Soare, Recursively enumerable sets and degrees, Springer-Verlag, 1987.

15. R. Watnick, A generalization of Tennenbaum's theorem on effectively finite recursive linear orderings, J. Symbolic Logic 49 (1984), 563-569.

(R. G. Downey) Department of Mathematics, Victoria University of Wellington, Wellington, New Zealand

Current address, M. F. Moses: Department of Mathematics, Western Illinois University, Macomb, Illinois 61455 